PROCEEDINGS OF THE

AMERICAN MATHEMATICAL SOCIETY

Volume 126, Number 9, September 1998, Pages 2705-2711

S $0002-9939(98) 04342-1$

\title{
CAUCHY-SCHWARZ AND MEANS INEQUALITIES FOR ELEMENTARY OPERATORS INTO NORM IDEALS
}

\author{
DANKO R. JOCIĆ
}

(Communicated by Palle E. T. Jorgensen)

Abstract. The Cauchy-Schwarz norm inequality for normal elementary operators

$$
\left\|\sum_{n=1}^{\infty} A_{n} X B_{n}\right\| \leq\left\|\left(\sum_{n=1}^{\infty} A_{n}^{*} A_{n}\right)^{1 / 2} X\left(\sum_{n=1}^{\infty} B_{n}^{*} B_{n}\right)^{1 / 2}\right\|,
$$

implies a means inequality for generalized normal derivations

$$
\left\|\frac{A X+X B}{2}\right\| \leq\|X\|^{1-\frac{1}{r}}\left\|\frac{|A|^{r} X+X|B|^{r}}{2}\right\|^{\frac{1}{r}},
$$

for all $r \geq 2$, as well as an inequality for normal contractions $A$ and $B$

$$
\left\|\left(I-A^{*} A\right)^{\frac{1}{2}} X\left(I-B^{*} B\right)^{\frac{1}{2}}\right\| \leq\|X-A X B\|,
$$

for all $X$ in $B(H)$ and for all unitarily invariant norms $\|\cdot\|$.

\section{INTRODUCTION}

Let $B(H)$ and $\mathcal{C}_{\infty}$ stand respectively for spaces of all bounded and all compact linear operators acting on a separable, infinite-dimensional, complex Hilbert space $H$. For an $X \in B(H)$ let $\|X\|$ denote its norm, and for an arbitrary $X \in \mathcal{C}_{\infty}$ let $s_{1}(X) \geq s_{2}(X) \geq \cdots$ denote the singular values of $X$, i.e., the eigenvalues of $|X|=\left(X^{*} X\right)^{1 / 2}$, arranged in a non-increasing order, with their multiplicities counted. Each "symmetric gauge function" $\Phi$ on sequences gives rise to a unitarily invariant (u.i.) norm on operators defined by $\|A\|_{\Phi}=\Phi\left(\left\{s_{n}(A)\right\}\right)$. We will denote by the symbol $\|\cdot\|$ any such norm. Any such norm is defined on a natural subclass $\mathcal{C}_{\|\cdot\|}$ of $\mathcal{C}_{\infty}$ called the norm ideal associated with the norm $\|\cdot\|$, and satisfies the invariance property $\|U A V\|=\|A\|$ for all $A$ in this ideal and for all unitary operators $U, V$. Each norm ideal $\mathcal{C}_{\|\cdot\|}$ is closed in the topology generated by the norm $\|\cdot\| \cdot$ Particularly well known among unitarily invariant norms are the Schatten $p$-norms, defined as $\|X\|_{p}=\left(\sum_{n=1}^{\infty} s_{n}^{p}(A)\right)^{1 / p}$ for $1 \leq p<\infty$ and $\|X\|_{\infty}=\|X\|=s_{1}(X)$, which represent the norms on the Schatten $p$-ideals $\mathcal{C}_{p}$. The Ky Fan norms, defined as $\|A\|_{k}=\Phi_{k}\left(s_{i}(A)\right)=\sum_{i=1}^{k} s_{i}(A)$ for $k=1,2, \cdots$, represent another interesting family of unitarily invariant norms. The associated ideals $\mathcal{C}_{\infty}^{(k)}$ consist of all compact operators as every Ky Fan $k$-norm is equivalent to the norm in $\mathcal{C}_{\infty}$. The property saying that for all $X \in \mathcal{C}_{\infty}$ and $Y \in \mathcal{C}_{\|\cdot\|}$ with $\|X\|_{k} \leq\|Y\|_{k}$ for all $k \geq 1$ we have

Received by the editors March 12, 1996 and, in revised form, February 4, 1997.

1991 Mathematics Subject Classification. Primary 47A30; Secondary 47B05, 47B10, 47B15.

Key words and phrases. Unitarily invariant norms, Ky Fan dominance property. 
$X \in \mathcal{C}_{\|\cdot\|}$ with $\|X\| \leq\|Y\|$ is known as the Ky Fan dominance property ([GK], ch. $3, \S 4)$. For a complete account of the theory of norm ideals, the reader is referred to $[\mathrm{GK}],[\mathrm{Sch}]$ and $[\mathrm{Si}]$.

If $\mathcal{A}=\left(A_{1}, \ldots, A_{N}\right)$ and $\mathcal{B}=\left(B_{1}, \ldots, B_{N}\right)$ are $N$-tuples of bounded Hilbert space operators, then the elementary operator $R=R_{\mathcal{A}, B}$ on $B(H)$ is defined by $R(X)=\sum_{n=1}^{N} A_{n} X B_{n}$. Elementary operators were introduced by Lumer and Rosenblum in [LR], who studied their spectral properties. In this setting many authors subsequently studied spectral, algebraic, metric and structural properties of elementary operators (see [F83], [F85], [F87], [FL], [McI], and the references therein).

If both $\left\{A_{n}\right\}_{n=1}^{N}$ and $\left\{B_{n}\right\}_{n=1}^{N}$ are families of commuting normal operators, one can easly show that the associated elementary operator $R_{\mathcal{A}, \mathcal{B}}$ is normal when restricted to the Hilbert space $\mathcal{C}_{2}$. So, in the sequel, by a normal elementary operator we will always mean such an operator, inluding those cases of $N=\infty$ with the convergence guaranted. As was shown by G. Weiss in his paper [W83], a famous Fuglede-Putnam type theorem extends to such operators. Very important examples of normal elementary operators are so called "pinching" operators $R_{\mathcal{P}}(X)=\sum_{n=1}^{\infty} P_{n} X P_{n}$, generated by a family of mutually orthogonal self-adjoint projections $\left\{P_{n}\right\}_{n=1}^{\infty}$.

\section{MAin RESUlts}

We start with the basic Cauchy-Schwarz norm inequality for normal elementary operators. The following theorem extends "pinching" theorems 2.5.1 of [GK] and 1.19 of $[\mathrm{Si}]$.

Theorem 2.1. If $\sum_{n=1}^{\infty} C_{n}^{*} C_{n} \leq 1, \sum_{n=1}^{\infty} C_{n} C_{n}^{*} \leq 1, \sum_{n=1}^{\infty} D_{n}^{*} D_{n} \leq 1$ and $\sum_{n=1}^{\infty} D_{n} D_{n}^{*} \leq 1$ for some operator families $\left\{C_{n}\right\}_{n=1}^{\infty}$ and $\left\{D_{n}\right\}_{n=1}^{\infty}$, then also $\sum_{n=1}^{\infty} C_{n} Y D_{n} \in \mathcal{C}_{\|\cdot\| \cdot \|}$ whenever $Y \in \mathcal{C}_{\|\cdot\|}$ for some unitarily invariant norm $\|\cdot\|$, and moreover

$$
\left\|\sum_{n=1}^{\infty} C_{n} Y D_{n}\right\| \leq\|Y\| .
$$

Proof. For arbitrary $f$ and $g$ in $H$ a straightforward calculation gives

$$
\begin{aligned}
& \left|\left\langle\left(\sum_{n=1}^{\infty} C_{n} Y D_{n}\right) f, g\right\rangle\right| \leq \sum_{n=1}^{\infty}\|Y\|\left\|D_{n} f\right\|\left\|C_{n}^{*} g\right\| \\
& \quad \leq\|Y\|\left(\sum_{n=1}^{\infty}\left\|D_{n} f\right\|^{2}\right)^{1 / 2}\left(\sum_{n=1}^{\infty}\left\|C_{n}^{*} g\right\|^{2}\right)^{1 / 2} \\
& \quad=\|Y\|\left\langle\sum_{n=1}^{\infty} D_{n}^{*} D_{n} f, f\right\rangle^{1 / 2}\left\langle\sum_{n=1}^{\infty} C_{n} C_{n}^{*} g, g\right\rangle^{1 / 2} \\
& =\|Y\|\left\|\left(\sum_{n=1}^{\infty} C_{n} C_{n}^{*}\right)^{1 / 2} g\right\|\left\|\left(\sum_{n=1}^{\infty} D_{n}^{*} D_{n}\right)^{1 / 2} f\right\| \leq\|Y\|\|f\|\|g\|,
\end{aligned}
$$

from which we conclude that

$$
\left\|\sum_{n=1}^{\infty} C_{n} Y D_{n}\right\| \leq\|Y\| .
$$


Therefore, for all $N=1,2, \cdots$, for $Y \in \mathcal{C}_{1}$ and for all $W \in B(H)$ we have

$$
\begin{aligned}
& \left|\operatorname{tr}\left(\sum_{n=1}^{N} C_{n} Y D_{n} W^{*}\right)\right|=\left|\operatorname{tr}\left(Y\left(\sum_{n=1}^{N} C_{n}^{*} W D_{n}^{*}\right)^{*}\right)\right| \\
& \quad \leq\|Y\|_{1}\left\|\sum_{n=1}^{N} C_{n}^{*} W D_{n}^{*}\right\| \leq\|Y\|_{1}\|W\|,
\end{aligned}
$$

according to (2.2), from which we deduce that

$$
\left\|\sum_{n=1}^{N} C_{n} Y D_{n}\right\|_{1} \leq\|Y\|_{1} .
$$

If $Y \in \mathcal{C}_{\infty}$, let $Y=\sum_{n=1}^{\infty} s_{n}(Y)\left\langle\cdot, e_{n}\right\rangle f_{n}$ be a singular value decompositon for some orthonormal systems $\left\{e_{n}\right\}$ and $\left\{f_{n}\right\}$. For all $k \geq 2$ we introduce operators $Z=\sum_{n=1}^{k-1}\left(s_{n}(Y)-s_{n+1}(Y)\right) \sum_{j=1}^{n}\left\langle\cdot, e_{j}\right\rangle f_{j}$ and $V=s_{k}(Y) \sum_{n=1}^{k}\left\langle\cdot, e_{n}\right\rangle f_{n}+$ $\sum_{n=k+1}^{\infty} s_{n}(Y)\left\langle\cdot, e_{n}\right\rangle f_{n}$. We see that

$$
\begin{aligned}
Z & =\sum_{n=1}^{k-1} \sum_{j=1}^{n}\left(s_{n}(Y)-s_{n+1}(Y)\right)\left\langle\cdot, e_{j}\right\rangle f_{j} \\
& =\sum_{j=1}^{k}\left(s_{j}(Y)-s_{k}(Y)\right)\left\langle\cdot, e_{j}\right\rangle f_{j} \\
& =\sum_{n=1}^{k} s_{n}(Y)\left\langle\cdot, e_{n}\right\rangle f_{n}+s_{k}(Y) \sum_{n=1}^{k}\left\langle\cdot, e_{n}\right\rangle f_{n}=Y-V .
\end{aligned}
$$

We can also note that $s_{1}(V)=\cdots=s_{k}(V)=s_{k}(Y)$, due to orthogonality of the systems $\left\{e_{n}\right\}$ and $\left\{f_{n}\right\}$. That will allows us to conclude that for all Ky Fan $k$-norms we have

$$
\begin{aligned}
& \left\|\sum_{n=1}^{N} C_{n} Y D_{n}\right\|\left\|_{k} \leq\right\| \sum_{n=1}^{N} C_{n} Z D_{n}\|\|_{k}+\left\|\sum_{n=1}^{N} C_{n} V D_{n}\right\| \|_{k} \\
& \quad \leq\|Z\|_{1}+k\left\|\sum_{n=1}^{N} C_{n} Z D_{n}\right\|_{\infty} \\
& \leq \sum_{n=1}^{k-1}\left(s_{n}(Y)-s_{n+1}(Y)\right) \sum_{j=1}^{n}\left\|\left\langle\cdot, e_{j}\right\rangle f_{j}\right\|_{\infty}+k\|V\|_{\infty} \\
& \leq \sum_{n=1}^{k-1} n\left(s_{n}(Y)-s_{n+1}(Y)\right)+k s_{k}(Y)=\sum_{n=1}^{k} s_{n}(Y)=\|Y\|_{k},
\end{aligned}
$$

with (2.4) following from (2.3) and (2.5) from (2.2).

Moreover, if $Y$ is in $\mathcal{C}_{\infty}$ then also $\sum_{n=1}^{\infty} C_{n} Y D_{n} \in \mathcal{C}_{\infty}$. Indeed, elementary operators $R_{N}(Y)=\sum_{n=1}^{N} C_{n} Y D_{n}$ acting on $\mathcal{C}_{\infty}^{(k)}$ represent a bounded family, because $\left\|R_{N}(Y)\right\|_{k} \leq\|Y\|_{k}$ for all $Y \in \mathcal{C}_{\infty}$ by (2.6). Also, for one dimensional operators 
$f \otimes g$ and $M>N$ we have

$$
\begin{aligned}
\left\|R_{M}(f \otimes g)-R_{N}(f \otimes g)\right\|_{k} & \leq\left\|\sum_{n=N+1}^{M} D_{n}^{*} f \otimes C_{n} g\right\|_{1} \\
\leq \sum_{n=N+1}^{M}\left\|D_{n}^{*} f\right\|\left\|C_{n} g\right\| & \leq\left\|\left(\sum_{n=N+1}^{M} C_{n} C_{n}^{*}\right)^{\frac{1}{2}} g\right\|\left\|\left(\sum_{n=N+1}^{M} D_{n}^{*} D_{n}\right)^{\frac{1}{2}} f\right\|,
\end{aligned}
$$

which $\longrightarrow 0$ as $M, N \longrightarrow \infty$. Therefore $R_{N}(Y)$ converge in $\mathcal{C}_{\infty}^{(k)}$ for all finite dimensional $Y$ to a compact operator. By the uniform boundedness principle the same is true for all $Y \in \mathcal{C}_{\infty}^{(k)}$, due to its separability. So (2.1) holds for all Ky Fan $k$-norms, and we therefore invoke the Ky Fan dominance property to conclude that (2.1) holds for all unitarily invariant norms, as required.

In the sequel we will refer to a family $\left\{A_{n}\right\}_{n=1}^{\infty}$ in $B(H)$ as square summable if $\sum_{n=1}^{\infty}\left\|A_{n} f\right\|^{2}<\infty$ for all $f \in H$. Though this means just the weak convergence of $\sum_{n=1}^{\infty} A_{n}^{*} A_{n}$, an appeal to the resonance principle shows that $\sum_{n=1}^{\infty} A_{n}^{*} A_{n}$ actually defines a bounded Hilbert space operator, and due to the monotonicity of its partial sums, the convergence is moreover strong. For such families the following Cauchy-Schwarz inequality holds:

Theorem 2.2. For a square summable families $\left\{A_{n}\right\}_{n=1}^{\infty}$ and $\left\{B_{n}\right\}_{n=1}^{\infty}$ of commuting normal operators

$$
\left\|\sum_{n=1}^{\infty} A_{n} X B_{n}\right\| \leq\left\|\left(\sum_{n=1}^{\infty} A_{n}^{*} A_{n}\right)^{1 / 2} X\left(\sum_{n=1}^{\infty} B_{n}^{*} B_{n}\right)^{1 / 2}\right\|,
$$

for all $X \in B(H)$ and for all u.i. norms $\|\cdot\|$. If $\mathcal{C}_{\|\cdot\| \|}$ is separable and $X \in \mathcal{C}_{\|\cdot\| \cdot \|}$, then the left-hand side sum converges in the norm of this ideal.

Proof. First, we need a suitable factorization for Hilbert space operators $A_{n}$ and $B_{n}$. Let $A=\left(\sum_{n=1}^{\infty} A_{n}^{*} A_{n}\right)^{\frac{1}{2}}$ and $B=\left(\sum_{n=1}^{\infty} B_{n}^{*} B_{n}\right)^{\frac{1}{2}}$, and let $P$ and $Q$ denote respectively the orthogonal projections on $\overline{R(A)}$ and $\overline{R(B)}$. If for a given $f \in H$ we have that $P f=\lim _{k \longrightarrow \infty} A g_{k}$ for some sequence $\left\{g_{k}\right\}$ in $H$, then $\lim _{k} \longrightarrow \infty A_{n} g_{k}$ exists for all $n \geq 1$ and does not depend on the chosen sequence. Indeed,

$$
\left\|A_{n} g_{k}-A_{n} g_{l}\right\| \leq\left\|A\left(g_{k}-g_{l}\right)\right\| \longrightarrow\|P f-P f\|=0
$$

as $k, l \longrightarrow \infty$, and also $\left\|A_{n} g_{k}-A_{n} h_{k}\right\| \leq\left\|A\left(g_{k}-h_{k}\right)\right\| \longrightarrow 0$ as $k \longrightarrow \infty$ whenever $\lim _{k \rightarrow \infty} A h_{k}=P f$ for some other sequence $\left\{h_{k}\right\}$. Thus we can correctly introduce operators $C_{n}, n=1,2, \cdots$, by $C_{n} f=\lim _{k \rightarrow \infty} A_{n} g_{k}$, where $\left\{g_{k}\right\}$ is any sequence in $H$ such that $\lim _{k \rightarrow \infty} A g_{k}=P f$. Let us note that due to our definition every $C_{n}$ vanishes on $N(A)$, i.e., $C_{n}=C_{n} P$, and also $C_{n} A=A C_{n}=A_{n}$. Moreover, $\sum_{n=1}^{\infty} C_{n}^{*} C_{n}=P$. Indeed, $\sum_{n=1}^{\infty} C_{n}^{*} C_{n} A^{2}=\sum_{n=1}^{\infty} A_{n}^{*} A_{n}=A^{2}$ implies $\sum_{n=1}^{\infty} C_{n}^{*} C_{n} P=P$, which together with the fact that $C_{n}(I-P)=0$ gives the desired conclusion. For all $m, n=1,2, \cdots, C_{m}^{*}$ and $C_{n}$ comute on $R\left(A^{2}\right)$ and $N\left(A^{2}\right)$, and so also on all of $H$. Thus $\left\{C_{n}\right\}_{n=1}^{\infty}$ is a commuting family of normal contractions which realize the factorizations $C_{n} A=A C_{n}=A_{n}$, with $\sum_{n=1}^{\infty} C_{n}^{*} C_{n}=P$, and which commute with the family $\left\{A_{n}\right\}_{n=1}^{\infty}$. Similarly we get a commuting family $\left\{D_{n}\right\}_{n=1}^{\infty}$ of normal contractions which also commute with $\left\{B_{n}\right\}_{n=1}^{\infty}$ and satisfy $D_{n} B=B D_{n}=B_{n}$ and $\sum_{n=1}^{\infty} D_{n}^{*} D_{n}=Q$. One could easily derive the next explicit 
formula: $C_{n}=\overline{A_{n} A^{\dagger}}=\overline{A^{\dagger} A_{n}}$, where $A^{\dagger}$ denotes a (densely defined) Moore-Penrose (generalized) inverse for $A$.

For $Y=A X B \in \mathcal{C}_{\|\cdot\|}$ (there is nothing to prove in the opposite case), an application of Theorem 2.1 gives

$$
\begin{aligned}
& \left\|\sum_{n=1}^{\infty} A_{n} X B_{n}\right\|=\left\|\sum_{n=1}^{\infty} C_{n} Y D_{n}\right\| \\
& \leq\|Y\|=\left\|\left(\sum_{n=1}^{\infty} A_{n}^{*} A_{n}\right)^{1 / 2} X\left(\sum_{n=1}^{\infty} B_{n}^{*} B_{n}\right)^{1 / 2}\right\|,
\end{aligned}
$$

which proves the first part of theorem.

Finally, if $\mathcal{C}_{\|\cdot\|}$ is separable, then for all $N=1,2, \cdots$, an application of the just proven part of theorem combined with the arithmetic-geometric means inequality in $[\mathrm{BhD}]$ gives

$$
\begin{gathered}
\left\|\sum_{n=N}^{\infty} A_{n} X B_{n}\right\| \leq\left\|\left(\sum_{n=N}^{\infty} A_{n}^{*} A_{n}\right)^{\frac{1}{2}} X\left(\sum_{n=N}^{\infty} B_{n}^{*} B_{n}\right)^{\frac{1}{2}}\right\| \\
=\left\|\left(\sum_{n=N}^{\infty} C_{n}^{*} C_{n}\right)^{\frac{1}{2}} A X B\left(\sum_{n=N}^{\infty} D_{n}^{*} D_{n}\right)^{\frac{1}{2}}\right\| \\
\leq \frac{1}{2}\left\|\left(\sum_{n=N}^{\infty} C_{n}^{*} C_{n}\right) A X B+A X B\left(\sum_{n=N}^{\infty} D_{n}^{*} D_{n}\right)\right\| .
\end{gathered}
$$

We see by (2.8) that $\left\{\sum_{n=N}^{\infty} C_{n}^{*} C_{n}\right\}_{N=1}^{\infty}$ and $\left\{\sum_{n=N}^{\infty} D_{n}^{*} D_{n}\right\}_{N=1}^{\infty}$ represent bounded sequences of selfadjoint operators which strongly converge to 0 as $N \longrightarrow \infty$. As $A X B \in \mathcal{C}_{\|\| \cdot \|}$, which is separable, then the right-hand side of (2.9) tends to 0 as $N \longrightarrow \infty$ by Theorem 3.6.3. of [GK]. The conclusion follows.

Corollary 2.1. For normal $A$ and $B$ in $B(H)$ and for all real $r \geq 2$,

$$
\left\|\frac{A X+X B}{2}\right\| \leq\left\|\left(\frac{1+|A|^{r}}{2}\right)^{\frac{1}{r}} X\left(\frac{1+|B|^{r}}{2}\right)^{\frac{1}{r}}\right\|
$$

as well as

$$
\left\|\frac{X+A X B}{2}\right\| \leq\left\|\left(\frac{1+|A|^{r}}{2}\right)^{\frac{1}{r}} X\left(\frac{1+|B|^{r}}{2}\right)^{\frac{1}{r}}\right\|
$$

for all $X \in B(H)$ and for all u.i. norms $\|\cdot\| \cdot$

Proof. $\{A, I\}$ and $\{I, B\}$ are families of normal commuting operators, and so for $r=$ 2 the desired conclusion follows by Theorem 2.2. For $r>2$ the mapping $t \longrightarrow t^{\frac{2}{r}}$ is operator monotone by a well known Heinz therem, and therefore this is an operator concave mapping (see $[\mathrm{BSh}]$ ). Specifically, $\frac{1+|A|^{2}}{2} \leq\left(\frac{1+|A|^{r}}{2}\right)^{\frac{2}{r}}$, from which we obtain $\left\|\left(\frac{1+|A|^{2}}{2}\right)^{\frac{1}{2}}\left(\frac{1+|A|^{r}}{2}\right)^{-\frac{1}{r}}\right\| \leq 1$ and similarly $\left\|\left(\frac{1+|B|^{2}}{2}\right)^{\frac{1}{2}}\left(\frac{1+|B|^{r}}{2}\right)^{-\frac{1}{r}}\right\| \leq 1$. Therefore

$$
\left\|\left(\frac{1+|A|^{2}}{2}\right)^{\frac{1}{2}} X\left(\frac{1+|B|^{2}}{2}\right)^{\frac{1}{2}}\right\| \leq\left\|\left(\frac{1+|A|^{r}}{2}\right)^{\frac{1}{r}} X\left(\frac{1+|B|^{r}}{2}\right)^{\frac{1}{r}}\right\|,
$$

which completes the proof. 
Corollary 2.2. For normal $A$ and $B$ in $B(H)$ the inequality

$$
\left\|\frac{A X+X B}{2}\right\| \leq\|X\|^{1-\frac{1}{r}}\left\|\frac{|A|^{r} X+X|B|^{r}}{2}\right\|^{\frac{1}{r}}
$$

holds for all real $r \geq 2$, for all u.i. norms $\|\cdot\|$ and for all $X \in \mathcal{C}_{\|\cdot\| \cdot}$.

Proof. By Corollary 2.1, for all $t>0$,

$$
\begin{aligned}
& \left\|\frac{A X+X B}{2}\right\|=t^{-1}\left\|\frac{t A X+X t B}{2}\right\| \| \\
& \leq t^{-1}\left\|\left(\frac{1+|t A|^{r}}{2}\right)^{\frac{1}{r}} X\left(\frac{1+|t B|^{r}}{2}\right)^{\frac{1}{r}}\right\|,
\end{aligned}
$$

and therefore

$$
\left\|\frac{A X+X B}{2}\right\| \leq t^{-1}\|X\|^{1-\frac{2}{r}}\left\|\left(\frac{1+|t A|^{r}}{2}\right)^{\frac{1}{2}} X\left(\frac{1+|t B|^{r}}{2}\right)^{\frac{1}{2}}\right\|^{\frac{2}{r}},
$$

by [Ki], because $\frac{2}{r}<1$. Therefore, the arithmetic-geometric mean inequality implies

$$
\begin{gathered}
\left\|\frac{A X+X B}{2}\right\| \leq \frac{1}{2 t}\|X\|^{1-\frac{2}{r}}\left\|\frac{1+|t A|^{r}}{2} X+X \frac{1+|t B|^{r}}{2}\right\| \|^{\frac{2}{r}} \\
\leq \frac{1}{2}\|X\|^{1-\frac{2}{r}}\left(t^{-\frac{r}{2}}\|X\|+t^{\frac{r}{2}}\left\|\frac{|A|^{r} X+X|B|^{r}}{2}\right\|\right)^{\frac{2}{r}} .
\end{gathered}
$$

As the right-hand side equals $\|X\|^{1-\frac{1}{r}}\left\|\frac{|A|^{r} X+X|B|^{r}}{2}\right\|^{\frac{1}{r}}$, which attains its minimum for $t=\|X\|^{\frac{1}{r}}\left\|\frac{|A|^{r} X+X|B|^{r}}{2}\right\|^{-\frac{1}{r}}$, the conclusion follows.

Theorem 2.3. For normal contractions $A$ and $B$ the inequality

$$
\left\|\left(I-A^{*} A\right)^{\frac{1}{2}} X\left(I-B^{*} B\right)^{\frac{1}{2}}\right\| \leq\|X-A X B\|,
$$

holds for all $X \in B(H)$ and for all unitarily invariant norms $\|\cdot\| \cdot$

Proof. First, we note that $\mathrm{s}-\lim _{n \rightarrow \infty} A^{n}\left(I-A^{*} A\right)^{\frac{1}{2}}=0$. Indeed, by a spectral theorem, for every $f \in H$ there is a positive, finite Borel measure $\mu$ concentrated on $D=\{z \in \mathbf{C}:|z| \leq 1\}$ such that $\left\|A^{n}\left(I-A^{*} A\right)^{\frac{1}{2}} f\right\|^{2}=\int_{D}|z|^{2 n}\left(1-|z|^{2}\right) d \mu_{f}(z)$, whence the desired conclusion follows by Lebesgue's dominating convergence theorem. Therefore

$$
\text { w- } \lim _{n \longrightarrow \infty}\left(I-A^{*} A\right)^{\frac{1}{2}}\left(X-A^{n} X B^{n}\right)\left(I-B^{*} B\right)^{\frac{1}{2}}=\left(I-A^{*} A\right)^{\frac{1}{2}} X\left(I-B^{*} B\right)^{\frac{1}{2}} .
$$


So by Theorem 2.2 we get

$$
\begin{aligned}
\|(I & \left.-A^{*} A\right)^{\frac{1}{2}} X\left(I-B^{*} B\right)^{\frac{1}{2}} \| \\
& =\left\|\lim _{n \rightarrow \infty}\left(I-A^{*} A\right)^{\frac{1}{2}}\left(X-A^{n} X B^{n}\right)\left(I-B^{*} B\right)^{\frac{1}{2}}\right\| \| \\
& =\left\|\sum_{k=0}^{\infty}\left(I-A^{*} A\right)^{\frac{1}{2}} A^{k}(X-A X B) B^{k}\left(I-B^{*} B\right)^{\frac{1}{2}}\right\| \| \\
& \leq\left\|\left(\sum_{k=0}^{\infty}\left(I-|A|^{2}\right)|A|^{2 k}\right)^{\frac{1}{2}}(X-A X B)\left(\sum_{k=0}^{\infty}|B|^{2 k}\left(I-|B|^{2}\right)\right)^{\frac{1}{2}}\right\| \| \\
& =\|(I-P)(X-A X B)(I-Q)\| \leq\|X-A X B\|,
\end{aligned}
$$

where $P$ and $Q$ are the orthogonal projections on $\operatorname{Ker}\left(I-A^{*} A\right)$ and $\operatorname{Ker}\left(I-B^{*} B\right)$ respectively. This concludes the proof.

\section{REFERENCES}

[BSh] J. Bendat and S. Sherman, Monotone and Convex Operator Functions, Trans. Amer. Math. Soc. 79 (1955), 58-71. MR 18:588b

[BhD] R. Bhatia and Ch. Davis, More matrix forms of the arithmetic-geometric mean inequality, SIAM J. Matrix. Anal. Appl. 14 (1993), 132-136. MR 94b:15017

[F83] L. Fialkow, Spectral properties of elementary operators, Acta Sci. Math. (Szeged), 46 (1983), 269-282. MR 85h:47003

[F85] L. Fialkow, Spectral properties of elementary operators II, Trans. Amer. Math. Soc. 290 (1985), 415-429. MR 86j:47005

[F87] L. Fialkow, The range inclusion problem for elementary operators, Michigan J. Math. 34 (1987), 451-459. MR 89a:47052

[FL] L. Fialkow and R. Loebl, Elementary mappings into ideals of operators, Illinois Journal Math. Vol 28 (1984), 555-578. MR 86g:47054

[GK] I.C. Gohberg and M.G. Krein, Introduction to the theory of linear nonselfadjoint operators, Transl. Math. Monographs, vol. 18, Amer. Math. Soc. Providence, R.I. 1969. MR 39:7447

[Ki] F. Kittaneh, Norm inequalities for fractional powers of positive operators, Lett. Math. Phys. 27 (1993), 279-285. MR 94e:47011

[LR] G. Lumer and M. Rosenblum, Linear operator equations, Proc. Amer. Math. Soc. 10 (1959), 32-41. MR 21:2927

[McI] A. McIntosh, A. Pryde and W. Ricker, Estimates for the solution of operator equation $\sum_{j=1}^{m} A_{j} Q B_{j}=U$, Operator Theory, Adv. \& Appl., Vol. 20, pp. 197-207. Birkhauser Verlag Basel 1988. MR 89k:47024

[Sch] R. Schatten, Norm Ideals of completely continuous operators, Springer-Verlag, Berlin, 1960. MR 22:9878

[Si] B. Simon, Trace Ideals and their applications, Cambridge University Press, 1979. MR 80k:47048

[W83] G. Weiss, An extension of the Fuglede commutativity theorem modulo the Hilbert-Schmidt class to the operators of the form $\sum M_{n} X N_{n}$, Trans. Amer. Math. Soc. 278 (1983), 1-20. MR 84e: 47026

University of Belgrade, Faculty of Mathematics, Studentski trg 16, P. O. Box 550, 11000 Belgrade, Yugoslavia

E-mail address: jocic@matf.bg.ac.yu 